\title{
Production Process Modelling Architecture to support improved Cyber-Physical Production Systems
}

\author{
Fabio Seixas-Lopes ${ }^{1,2}$, Jose Ferreira ${ }^{2}$, Carlos Agostinho ${ }^{2}$, Ricardo Jardim- \\ Goncalves $^{2}$ \\ ${ }^{1}$ Department of Electrical Engineering, FCT, NOVA University of Lisbon \\ 2829-516 Caparica, Portugal \\ fas.lopes@campus.fct.unl.pt \\ ${ }^{2}$ Centre of Technology and Systems, UNINOVA, \\ 2829-516 Caparica, Portugal \\ \{japf, ca, rg\}@uninova.pt
}

\begin{abstract}
With the proliferation of intelligent networks in industrial environments, manufacturing SME's have been in a continuous search for integrating and retrofitting existing assets with modern technologies that could provide low-cost solutions for optimizations in their production processes. Their willingness to support a technological evolution is firmly based on the perception that, in the future, better tools will guarantee process control, surveillance and maintenance. For this to happen, the digitalization of valuable and extractable information must be held in a cost-effective manner, through contemporary approaches such as IoT, creating the required fluidity between hardware and software, for implementing Cyber-Physical modules in the manufacturing process. The goal of this work is to develop an architecture that will support companies to digitize their machines and processes through an MDA approach, by modeling their production processes and physical resources, and transforming into an implementation model, using contemporary CPS and IoT concepts, to be continuously improved using forecasting/predictive algorithms and analytics.
\end{abstract}

Keywords: Cyber-Physical Systems, Internet of Things, Model Driven Architecture, Interoperability, Process Modelling, Artificial Intelligence

\section{Introduction}

The term cyber-physical systems (CPS) refers to intelligent systems with integrated computational, networking and physical capabilities [1]. In industrial production, the advantage of applying this paradigm to existing systems is to fill the gap between the cyber world, where data is exchanged and transformed, and the physical world in which we live [2]. By creating means to utilize data from pre-existing physical machinery and devices, every extractable information becomes a mean of observing 
the current processes, feeding different improvement possibilities and production mechanisms such as energy efficiency, waste management, resource planning, scheduling and process monitoring. These mechanisms have a meaningful impact to achieve financial, social and environmental benefits, especially in thriving SME's that wish to compete for larger economy markets while keeping a sustainable production [3].

Contemporarily, the CPS represent many opportunities and research challenges that include the design and development of next-generation airplanes and space vehicles, high-end factories of the future, fully autonomous urban driving and prostheses that allow brain signals to control physical objects [4]. The focus of the concept is to expand the capabilities of the physical world through computation, communication and control, as key enablers for future technological developments.

In the physical world, unlike most of the software processes which are procedural, many things happen at once and are compositions of many parallel processes. In CPS, measuring and controlling the dynamics of such processes and using them in feedback loops that affect the computations is very important to orchestrate beneficial actions that influence their effectiveness. Consequently, concurrency is intrinsic in CPS. Many of the technical challenges in designing and analyzing embedded software stem from the need to bridge an inherently sequential semantics with an intrinsically concurrent physical world [5].

Considering that, this work tries to correspond to the following question: how can cyber-physical systems be improved using a model driven architecture such it is able to provide modular solutions/suggestions based on real-time data from the production processes?

In this work, the aim is to develop a simplified model driven architecture that allows the integration of modular optimization tools, that support the overall efficiency in the implementation of sustainable CPS in the manufacturing industry. The idea is to support existing SME's with a cheap but scalable solution for technological improvement. For this to be sustainable, the whole process must be analyzed, from the input of raw materials to the output of finished products.

With business notation (e.g. Business Process Model Notation (BPMN)) and the creation of data-driven models that represent the current production processes, a digital twin of the factory can be created and fed using real-time data. This data can be acquired using current cost-effective methodologies such as presented in [6] to retrofit any machinery and devices. The use of wearables on factory workers can also be regarded in a way that supports security and safety methodologies (e.g. insoles that measure weight and textiles to measure posture). The conception of such models allows for the creation of a decision support system that considers existing KPIs (Key Performance Indicators) to suggest possible improvements. These suggestions are based on AI (Artificial Intelligence) and machine-learning techniques, with forecasting/predictive analytics that dynamically adapt to the real-time acquired data (big data algorithms and complex event processing (CEP) methodologies will be used).

Thus, this work proposes an architecture that is able to consider the manufacturing ecosystem and create a decision support system that not only displays valuable information to management personnel but also actively thrives to propose improvements that, ideally, were proven to be successful in other implementations, or 
during validation. The idea is for the architecture to be translated into a secure platform, where the main blocks are the manufacturing resources and their specifications (machinery, materials, devices and people), the process models (it also includes the devices configurations), the enterprise system (factory production orders, the legacy ERP and other existing software that outputs valuable information), the data management system (real-time data acquisition and handling, CEP, DB's for resources, production orders and process models), the decision support block (with KPI's, AI, validated optimization tools and other testing community tools) and the process orchestrator (with a process engine, a process interface for monitorization and for the user to handle the production decision points and an optimization interface to manage the implemented optimization tools). These blocks must be fully interoperable to allow the introduction of any information source to be valuable to this architecture. In this way, any information source is also modular, not strictly necessary, but valuable for the performance, even if they are redundant (in case of malfunctioning, see [7]). The implementation of information sources and their location/specifications (what types of sensors, where they are placed considering the type of industry, etc.) is also something that could be suggested by the decision support system when it may result in optimization.

From the factory's management perspective, the modus operandi is the initial specification of the production processes and resources in the platform, to enable the creation of a digital model. After that, the data management block is populated with information, that can be complemented with the connection of the enterprise system block (to use legacy software and existing production orders), and the process orchestrator is able to provide a monitorization and data visualization, regarding the actual production processes. With this, the main blocks of the architecture are in place and the tools for process improvement are ready to be of value. By matching KPI's and AI technology, the decision support block is able to suggest and pre-configure tools based on real-time data from the processes. The tools can be modular and added during time, considering cost and other constraints.

The direction of this work is to have a simplified solution for companies to digitize their assets, build an implementation model, have production process monitorization and quickly act on acquired information, resulting in performance improvements.

\section{Relationship to Tech Innovation for Life Improvement}

Digital innovation is a requisition for industrial improvement but to be sustainable, on a business but also on an environmental level, solutions must be planned and considered within a scalable and responsible paradigm. Considering the contemporary industry and the respective environmental impacts, it is very important to define sustainable mechanisms along with the optimization of manufacturing processes. Usually, the implementation of such mechanisms can disrupt the existing functioning of factories, which imposes the necessity of not repeatedly trying to implement new solutions. Considering that, an architecture that dynamically envisions environmental sustainability, from the start, as a mean to pursuit economic growth by reducing costs, can be very appealing and beneficial by not defining specific procedures that may 
need to be replaced in the future. This impacts the environmental footprint but also the stability of companies, specially SME's which then are more at easy to provide better working conditions and stable jobs for their workers. Also, people are regarded in this architecture from the start, which enables the application of security and safety methodologies (e.g. acquiring wearables) as optimization tools.

\section{Background}

To specify and better understand the methodology proposed in this paper, some key concepts will be mentioned and explained. The objective is to provide a brief insight on the concepts that generate the modules proposed in the next section.

IoT and Data Acquisition. The Internet of Things (IoT) is an emerging topic that aims to combine consumer products, sensors and industrial components and other everyday objects with Internet connectivity and powerful data analytic capabilities that have the ability to transform the way we work and live [8]. With this, objects can become optimized, as every extractable information becomes a mean of analyzing and computing the functioning processes. The results of such analysis are oriented to provide better performance, enhanced context functioning and new purposes that come into play when connecting objects to an intelligent network [7].

For the purpose of this work, IoT represents the ideal conception of data acquisition within the SME's industrial environment, where data sources tend to provide heterogenous data, if there are any in place. It can be a cost-effective solution of implementing intelligent network nodes that pre-filter data, considering context, and pass on valuable information for decision-making processes on higher levels of the architecture [9]. Depending on how this concept is envisioned, the data acquisition process can also consider existing knowledge bases, data/event handlers and other mechanisms that contribute to a better understanding of data and how it can be handled, considering objectives, context, incorrect input and fault detection.

Cyber-Physical Systems. This concept was briefly defined in the introduction section of this work as a mean to connect the digital world to the physical world. Its importance stems from the necessity to have a digital form of acquirable physical information that can be quickly displayed and analyzed, i.e. a digital twin [10].

This methodology allows to monitor production as a whole and to plan and model optimization based on the acquired information. In other words, by designing models that represent the manufacturing process and connect them to the acquisition modules, there is space for designing a runtime solution that implements mechanisms such as scheduling, waste and energy consumption/efficiency, that result in direct optimization of processes and also contribute for more complex decision-making processes that need a global overview of data and resources in order to be effective.

In this work, CPS conceptually represents the environment for the purposed architecture, since the idea is to have real-time information, fed by gradually implementable information sources, to develop sustainable optimization mechanisms and contribute to an intelligent and dynamic decision-making system. 
Process Modelling and Model-Driven Architectures. The process modelling process can be described as a design procedure to reinforce or create methodologies for enabling mechanisms such as simulation and production monitorization, and in this case the implementation or improvement of a CPS and production processes, by creating a digital twin [11]. This is where the user's knowledge gains shape to result in a representation of the factories resources and production processes.

A Model-Driven Architecture puts the process models in the center of the software development process and it is driven by the activity of these models [12]. The models typically derive from requirements, which are subject to analysis, followed by lowlevel design, the design itself, testing and deployment.

The proposed architecture of this work is heavily model-driven and for making sense of the proposed modules, the specification of the assets is crucial to have a real understanding of the optimization possibilities. The flow of the production is as essential too, because it delineates how the process occurs, which steps can provide feedback, when do materials are needed and transformed, when mechanisms of maintenance, scheduling and other strategic planning situations may occur, etc.

\section{Production Process Modelling Architecture}

In this section, an overview of the general architecture for this work is proposed. It is important to mention that this architecture is currently based on a process modelling architecture that envisions the application of possible complex technological processes (AI, machine-learning, big data, etc.) that may induce alterations along its design and the continuity of this work. Also, guidelines from area standard architectures/models/ontologies/frameworks such as ARROWHEAD, IoT-A, W3C SSN Ontology and IoT Lite, were considered to develop this work, with the objective of developing a real-world integrable and interoperable solution.

This work also considers previous work and early results obtain, within the scope of IoT and CPS, by the authors of this paper in ongoing and finished European research projects, for the purpose of designing this architecture's modules and how they are envisioned to be interoperable.

\subsection{Architecture's Overview}

A high-level view of the architecture proposed in this work is presented in Fig.1.

The main idea for this architecture, in addition to each module functionalities, is the interoperability that is aimed to be achieved during the runtime phase by combining the functionalities of a process orchestrator engine with incoming realtime data to provide production monitorization, decision points, user input and data visualization, and the decision support system which is fed with the information handled in the process orchestrator and compares it with previously obtained knowledge (from assets, models, type of industry and materials, and other implementations) stored in specific databases to suggest valuable improvements that can be the implementation of optimizing modules (e.g. capacity planner, scheduler 
engine, process simulation), alterations in specific points of the production process, addition of sensors or data sources, re-configuration of resources, analyzing the costbenefit ratio for upgrading machinery or suggesting to hire more workers, etc.

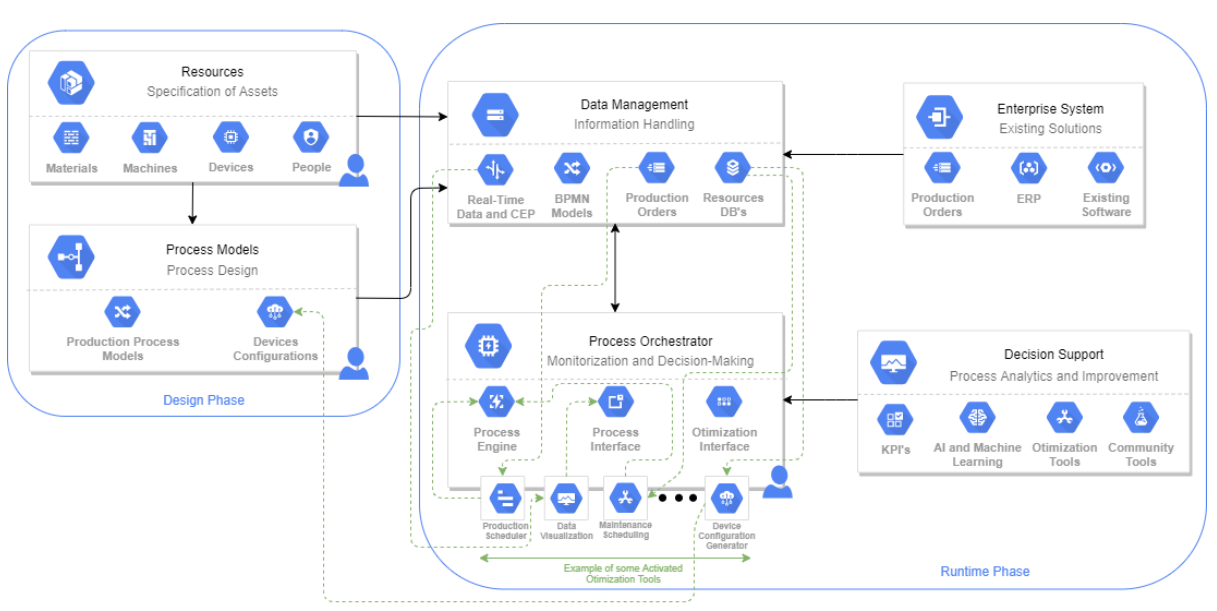

Fig. 1. General overview of the proposed architecture with examples of an implementation of some optimization modules.

\subsection{Architecture's Blocks}

In this sub-section, a brief description of the main blocks of this architecture is given, to explain the main functionalities and support their interaction and interoperability.

Resources and Process Models. For enabling the architecture with real-time data, the production assets must be specified. This specification is very important not only to establish data sources, through IoT nodes and hubs, but also to empower the decision support system with possibilities regarding the characteristics of machinery, sensors, actuators, and other production assets.

After the assets are digitalized, the overall production flow is created and connected to the data sources, so it can enable the monitorization of the production processes in real-time with live input of collected data.

For this to be optimized, the methodologies implied by following an MDA approach are applied to implement the designing of models (e.g. using BPMN) based on the process requirements and subsequent analysis. For this, a low-code programming tool, such as Node-RED, can be used wire together the IoT implementation. Within the models, the sensors and their characteristics are very important because it allows to combine them with the mentioned requirements to transformed them in actual code to be used in the devices. With this, the IoT nodes and hubs implementation is faster and it can always be re-configured by higher-level blocks, since these models and configurations are stored in the data management block, that is accessible by the process orchestrator. 
Once these processes are finished, the data management and decision support blocks are engaged, and the user interacts with an interface. Further inclusion of additional data sources can or not require the adaptation of the process models, depending on how it can change the production flow (e.g. if creates a decision point).

Enterprise System. Already implemented mechanisms for the factory functioning must be used to not totally disrupt operations. Production orders and the output from the ERP (Enterprise Resource Planning) and other existing software are fed into the data management block to be stored and used by the process orchestrator.

Data Management. Acquired information, from the various data sources defined in the production resources, moves from phase to phase and needs to be managed in order to provide important contextual value. This includes mechanisms such as data filtering, fault detection, event processing and other data management processes, that ensure the real-time monitorization carried out by the process orchestrator.

Within properly structed databases, information about the production models, resources and the production orders are kept during reasonable durations of time.

Decision Support System. This block considers the company's existing knowledge bases (for the assets, type of industry, etc.), correlates them with the production assets and process models using forecasting/predictive analytics and algorithms, to create suggestions for improving the current production processes. These improvements may be based on the data source configuration, implementation of modules such as a raw material planner, creation/deletion of decision points in the production flow, etc.

The idea that motivates this system is to provide a dynamic approach for improvement and adaptation of production processes, creating an additional layer within the conventional CPS architectures.

Process Orchestrator. The purpose of this block is to provide an interface for the monitorization of the production processes and to enable a platform for decisionmaking. Here, is where the user gets process improvement suggestions (originated by the decision support block) through an optimization interface.

The Process Orchestrator runs on process engine and enables the inclusion of already developed and pre-configured modular tools such as scheduling, resource planning, device configuration generator. These modules, once activated, can be finetuned with more advanced configurations, and run inside the process orchestrator, serving specific functions and providing feedback for the user.

\section{Conclusions and Future Work}

This work provides an architecture for enhancing CPS with process modelling that considers all the production processes that transform the raw materials into the final products. Architectural structures to support optimizations were defined, on the data acquisition (combining them with models), data management, process monitorization and decision-making, to create an environment that allows step by step optimization, 
that will use predictive and forecasting algorithms that suggest the suitability for each production case. This approach follows current tendencies for technological development, using potentially complex concepts such as AI to dynamically adapt to real-time data and allocate the data analysis to processors and away from the user, which interacts with graphical interfaces that allow the drag-and-drop of features.

In the future, the proposed architecture is going to be validated using real-time data from industry to determine how the decision support block reacts and improves during time. The inherent technological concepts to achieve this are going to be included step by step, to ensure functionality and proper validation of their usefulness. Other industry standard methodologies and frameworks will serve as guidelines to ensure proper real-world integration and interoperability.

The specifications of production resources and the resulting process models are major tasks for ensuring the functionalities of this architecture which the degree of specification is something that is being currently validated, so it can reach a sustainable ratio of effort-benefit to the user.

The process orchestrator validation relies on previous work from this paper authors that pursued the implementation of an orchestrator based on BPMN process modeling and engine. Future work on this will be to develop this orchestrator as a cloud-enabled solution to include modular optimization tools. An interface for the user for decisionmaking, a graphical visualization of data and process monitorization, are also important achievements to validate this architecture for future use-case scenarios.

\section{References}

1. R. Baheti and H. Gill, "Cyber-physical Systems: The Impact of Control Technology," IEEE Control Syst. Soc., 2011.

2. J. Ferreira, F. Lopes, S. Ghimire, G. Doumeingts, C. Agostinho, and R. Jardim-Goncalves, "Cyber-Physical Production Systems to Monitor the Polishing Process of Cutlery Production," 9th Int. Conf. Intell. Syst. 2018 Theory,

3. A. Moeuf, R. Pellerin, S. Lamouri, S. Tamayo-Giraldo, and R. Barbaray, "The industrial management of SMEs in the era of Industry 4.0," Int. J. Prod. Res., 2018.

4. R. Baheti and H. Gill, Cyber-Physical Systems: From Theory to Practice. 2011.

5. S. A. Lee, E. A., \& Seshia, Introduction to Embedded Systems. A Cyber-Physical Systems Approach. Second Edition. 2017.

6. C. Agostinho, F. Lopes, J. Ferreira, S. Ghimire, and M. Marques, "A lightweight IoT hub for SME manufacturing industries," Proc. I-ESA Conf., vol. 9, pp. 371-383, 2019.

7. F. Lopes, J. Ferreira, R. Jardim-Goncalves, and C. Agostinho, "Semantic maps for IoT network reorganization in face of sensor malfunctioning," 2017 IEEE Int. Conf. Syst. Man, Cybern. SMC 2017, vol. 2017-Janua, pp. 1914-1919, 2017.

8. K. Rose, S. Eldridge, and L. Chapin, "THE INTERNET OF THINGS: AN OVERVIEW. Understanding the Issues and Challenges of a More Connected World.," Internet Soc., 2015.

9. Y. Huang and G. Li, "Descriptive models for Internet of things," in Proceedings of 2010 International Conference on Intelligent Control and Information Processing, ICICIP, 2010

10. T. H. J. Uhlemann, C. Lehmann, and R. Steinhilper, "The Digital Twin: Realizing the Cyber-Physical Production System for Industry 4.0," in Procedia CIRP, 2017.

11. B. Rodič, "Industry 4.0 and the New Simulation Modelling Paradigm," Organizacija, 2017.

12. A. Kleppe, et al., The Model Driven Architecture: Practice and Promise. 2003. 